\title{
FEATURE
}

\section{Can the dental team shape dietary behaviour?}

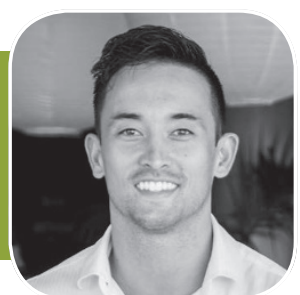

\section{Dr Steven Lin' examines the} expanding role of nutrition in oral healthcare.
So how can dental practices introduce more positive conversations around our teeth? For a start, there is a need for contemporary health messages to engage patients, instead of deterring them. Interestingly, a popular health topic in the media recently is the debate over the role of sugar in the chronic disease epidemic of obesity and type- 2 diabetes.

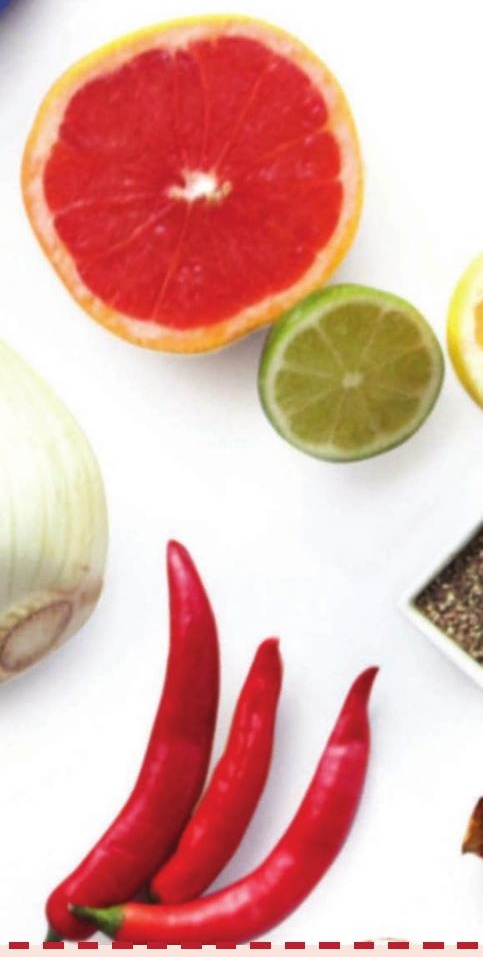

Food, the language of humanity There's no question people love food. Just take one look at the profiles of celebrities like Jamie Oliver and Gordon Ramsay and you'll see that food captures people's imagination.

Recently, cardiologist Aseem Malhotra has been heading calls in the media for a sugar tax and doctor Rangan Chatterjee has been featuring in his own show on the BBC outlining dietary approaches to medicine.

The dental industry has a history of trumpeting the cause of healthy eating. However, it took the popularity of TV Chef Jamie Oliver and his 'Sugar Rush' campaign to kick-start momentum towards a sugar tax in the UK. chore rather than an integral part of overall health.

Are oral health messages too negative? Twenty-five percent of adults in the UK haven't visited the dentist in the last two years. Dentists could be seen as too quick to scorn for not brushing properly or eating too many sweets. In either case, the choice to avoid the dental checkup is a detriment to oral health.

${ }^{7}$ Dr Steven Lin (www.drstevenlin.com) is a Sydney trained dentist, speaker and writer, currently working on his publication The Dental Diet. His twoday dental CPD course Nutrition and the Dental Practice will run on 14 and 15 October in Marble Arch, London. For tickets visit www.eventbrite.com.au/e/ london-nutrition-the-dental-practicetickets-26048855829.

\footnotetext{
'SUGAR IS A COMPLEX
TYPES ADDED TO
CHAIN. WHAT'S MOR
PRIMARY WEAKNESS
For the oral health profession there is a long established relationship between sugar intake and tooth decay. However, there needs to be an expansion of the scope of nutrition in the dental practice in order to advance the awareness of the mouth as a primary contributor to an overall preventive health approach.
}

The documentary featured the alarming rate of childhood decay leading to hospital admissions. Despite long educating about the established sugar and tooth decay relationship, the dental profession is often misheard when echoing the importance of oral and systemic health. 


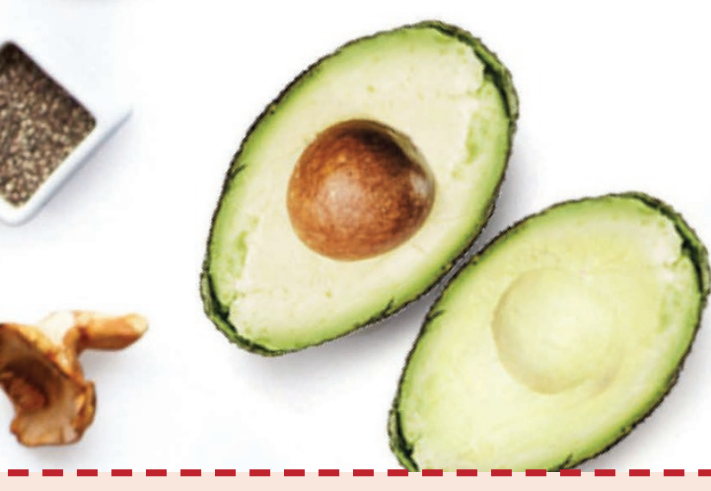

Why prevention begins with food Dietary behaviour represents a fundamental aspect of the oral disease paradigm. While dental professionals can beg until blue in the face to get people to brush and floss, the reality is less than a quarter of adults in the UK use dental floss regularly. However, while people will refuse to brush twice a day, they'll certainly continue to eat three times a day and dietary intake will always have a significant impact on dental disease risk.

The increasingly common chronic diseases facing society today including obesity, type2 diabetes and heart disease have all been heavily associated with lifestyle and diet. Similar to the sugar conversation, the dental profession was the first to communicate the correlation between diseases like periodontitis with heart disease and diabetes. However, remarkably absent from oral health practice is nutritional counsel that begins in the mouth with the aim to influence conditions all over the body.

The 'how to' guide for quitting sugar Eliminating sugar is certainly one of the primary goals that any dietary guidance for oral health should adhere to. But how does it work in application? Most have heard of the recommendation to reduce sugar consumption due to the relationship between sugar consumption and tooth decay. However, instructing patients to 'decrease the amount and frequency of sugar' without further nutritional leadership fails to acknowledge the difficulty of reducing sugar in the modern diet.
Sugar is a complex issue with many different types added to all levels of the food chain. What's more is it appeals to people's primary weakness for a sweet sensation. The result is a high level of confusion amongst consumers about the amount of sugar in their daily intake and the application of reducing sugar from one's diet. The dental surgery needs to expand its awareness and support infrastructure that provides practicable steps for people to change their health through sugar reduction via dietary replacement programmes.

Gut health begins in the mouth One of the hottest topics in healthcare today is the complex ro le of the human microbiome and 'gut health' in a variety of system and prevent diseases that begin with microbial imbalance.

Dietary programmes that establish a healthy oral microbiome and prevent oral disease also encourage better gut health. The mouth-gut connection is one of the simplest, yet most exciting ways that people can influence health outcomes all over their body and it all begins with a simple dietary change that can be implemented in the dental practice.

\section{Why dietary change needs a team approach}

Nutritional programmes allow the flow of positive interaction between not only the dental team and patients but also better communication of oral health messages to the broader community.

'OUTSIDE THE TREATMENT ROOM, THE DENTAL

\section{TEAM CAN PROVIDE NUTRITIONAL SUPPORT IN}

\section{THE FORM OF EDUCATIONAL SUPPORT, RECIPES}

\section{OR JUST A CONVERSATION ABOUT MEAL IDEAS.'}

common diseases. Digestive disorders like IBS, Crohn's disease and ulcerative colitis are slowly being understood as imbalances in the microbes that live within the digestive system.

However, the influence of microbes is extending beyond disorders of the digestive system to include conditions like food and skin allergies, diabetes and obesity. The healthcare profession faces a formidable challenge to sift through the mind-boggling complexity of the human microbiome to apply treatments to disease.

Tooth decay as a disease has long been understood as a disease mediated by bacteria. However, the traditional approach has been primarily focused on the elimination of harmful bacteria. What the human microbiome project taught us is that bacteria play a role in both health and disease. The key is to balance the populations that live within the digestive
For the dental practitioner, alongside diagnosis, it is imperative for lifestyle factors including food habits to be integrated into treatment communication. From here, dietary analysis can be performed by the practitioner and within a multi-disciplinary team that extends to a deeper understanding of disease risk and prevention.

However, expanding nutritional applications doesn't stop at the practitioner. Outside the treatment room, the dental team can provide nutritional support in the form of educational support, recipes or just a conversation about meal ideas.

With food in mind, the patient leaves the practice with a renewed impetuous to take control of their own oral health with a positive experience that will make sure they're calling back for their next dental appointment.

bdjteam 2016145 